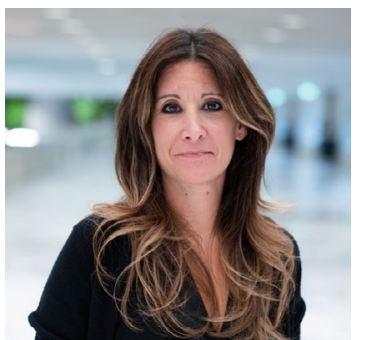

Credit: WHO / Chris Black

\title{
COVID-19 in 2022: controlling the pandemic is within our grasp
}

\author{
Vaccine inequity, inconsistent public health measures and new variants such as Omicron \\ are prolonging the COVID-19 pandemic, but controlling the virus remains possible.
}

A $s$ we enter the third year of the COVID-19 pandemic, public health leaders must take stock of where we are and how we can end the crippling hold that the SARS-CoV-2 virus has over us all. All pandemics end. The COVID-19 pandemic will end, but it is not over yet. Already, we have endured two years of missed opportunities, missed education, missed connections with family and loved ones. Without action, 2022 could be the same. But it doesn't have to be.

The virus will continue to affect our lives and livelihoods unless the global community collectively addresses inequitable access to vaccines, therapeutic agents and diagnostics, as well as the fact that we are giving the SARS-CoV-2 virus the room it needs to thrive through uneven and inconsistent national policies to reduce transmission, some of which are undermined by division and politicization. At the same time, governments must invest in preparedness, prevention and in science.

One of the greatest scientific achievements of the pandemic has been the speed of the development of several safe and effective COVID-19 vaccines. Robust data continue to show that COVID-19 vaccines are very effective at preventing people from getting seriously ill and dying. This protection seems to be maintained against the more transmissible Delta variant and over time.

However, we continue to see persistent inequities in access to COVID-19 vaccines. Of the more than 7 billion doses administered so far, less than $3 \%$ have been in countries on the African continent. The World Health Organization (WHO) has recommended that those at higher risk of severe disease and health workers be prioritised for COVID-19 vaccination in all countries. The number of doses administered by the end of September 2021 was enough to have covered $40 \%$ of the population in all countries. The failure to provide access of sufficient doses of vaccines to low- and middle-income countries is not only unethical, but it is epidemiologically and economically unwise, and is prolonging the pandemic.
Unfortunately, vaccines alone will not end this pandemic, in part because of more transmissible new variants and also because vaccines are primarily designed to protect against severe disease and death. The more the virus is allowed to circulate, the more opportunity the virus has to evolve. Throughout the course of the pandemic, SARS-CoV-2 has shown its ability to become better adapted to the human host, with variants Alpha and Delta demonstrating enhanced transmissibility. One of the biggest unknowns in 2022 will be how this evolution continues. Delta continues to evolve, and the Omicron variant has shown that the virus will continue to adapt, and such variants may be more transmissible, cause more or less severe disease, and/or develop properties of immune escape.

In 2022, epidemiological and genomic surveillance efforts should be expanded in all countries to detect SARS-CoV-2 variants and ensure that robust testing systems are linked to public health action. As we track the evolution of the virus, the WHO and partners will continue to closely assess and monitor the effect of virus evolution on public health and medical countermeasures, including diagnostics, therapeutic agents and COVID-19 vaccines.

Since the beginning of the pandemic, the WHO has recommended comprehensive measures to reduce SARS-CoV-2 transmission. Current vaccines by themselves are insufficient to stem transmission, and so increases in cases should be expected whenever public health and social measures are lifted, irrespective of vaccination coverage. In 2022, much of the world will need to continue with effective measures as we bring transmission under control. This includes, for example, wearing well-fitting masks, hand hygiene, physical distancing, improving ventilation of indoor spaces, avoiding crowded spaces and being supported to stay home if unwell.

It is essential to continue to improve national and sub-national public health infrastructure to better target and tailor local responses. Although the seasonal patterns of influenza and respiratory syncytial virus have been disrupted owing to the interventions for COVID-19, they have not disappeared from circulation, and governments should prepare for them to circulate again.

Preparedness for the next epidemic or pandemic pathogen does not begin when the current emergency is over. It begins now, with investing in integrated respiratory disease surveillance, a well-protected work force, early clinical care, access to health care, better personal protective equipment, further research and development for diagnostics, therapeutics and vaccines and addressing long-standing inequalities. This will not only help to control COVID-19, but also ensure that we are in a better position to confront the next outbreak.

In 2022, with increasing population level immunity, there will be substantial reductions in the number of people experiencing severe disease and death. This will alleviate some of the strain COVID-19 has exercised on even the most robust health-care systems. However, it is likely that there will be continued surges in transmission among unprotected individuals and strain on health-care systems in areas where vaccine coverage is low. The risk of more transmissible variants or variants with immune escape properties means that governments and communities must continue efforts to reduce transmission and protect the vulnerable, while slowly and carefully reopening our societies. Controlling this virus was always in our control, it remains in our control.

\section{Maria D. Van Kerkhove (ID) COVID-19 Health Operations and Technical Lead, Emerging Diseases and Zoonoses Unit Head, Health Emergencies Programme, World Health Organization, Geneva, Switzerland. \\ $\bigotimes_{e \text {-mail:vankerkhovem@who.int }}$}

Published online: 14 December 2021 https://doi.org/10.1038/s41591-021-01616-y 